\title{
latrogenic ureterovaginal fistula after laparoscopic hysterectomy: a case report
}

\author{
Jakub Marek Ratajczak ${ }^{1,2}$, Taras Hladun², Grzegorz Orchel ${ }^{2}$ \\ ${ }^{1}$ Department of Management and Logistics in Health Care, Medical University of Lodz, Lodz, Poland \\ 2Urology Department, Regional Specialized Hospital in Nowa Sól, Poland
}

\begin{abstract}
Introduction: The highest risk of intraoperative ureteral trauma is associated with hysterectomy, performed most frequently in postmenopausal women. The overall incidence of ureteral injuries varies in different studies between $0.5 \%$ and $10 \%$.

Case report: Ureterovaginal fistula following laparoscopic subtotal hysterectomy with bilateral salpingoophorectomy is reported in this case. Ureteral injury was not noticed during operation. Two weeks after the operation the patient noticed constant urine leakage from the vagina.

Discussion: A computed tomography scan revealed dilation of the left renal pelvis and the upper two thirds of the ureter due to an inflammatory fibrous mass with air bubbles involving its lower part. Contrast medium outflow identified the site of urine leakage. Subsequently, diagnostic cystoscopy and ureteroscopy revealed a fistula between the ureter and the apex of the vagina. The patient developed an iatrogenic ureterovaginal fistula, which was repaired successfully with a ureteroneocystostomy over a double-J stent a month and a half later. At the follow-up 3 months post operation there was no urine leakage from the vagina, no hydronephrosis in ultrasound check-up or ureterovaginal fistula on vaginal examination.

Conclusions: This paper highlights the problem of unnoticed ureteral injury during gynaecological surgeries, which, if overlooked, can develop into severe complications. Causes of ureteral injuries, prevention, and possible treatment options are also discussed.
\end{abstract}

Key words: ureter, vagina, injury, fistula, hysterectomy.

\section{Introduction}

Injury of the ureter is a risk in any pelvic or abdominal surgery, including laparoscopy and ureteroscopy, and it also can be a result of penetrating or blunt trauma. The overall incidence of ureteral injuries varies in different studies between $0.5 \%$ and $10 \%$ [1-7]. Gynaecological surgery remains the most common cause of iatrogenic ureteral injuries. The incidence of ureteral injuries is $0.1-1.5 \%$ in procedures due to benign diseases and rises to $5 \%$ in surgery of malignancies in gynaecology. $30-45 \%$ of injuries are diagnosed intraoperatively, and $55-70 \%$ of injuries are diagnosed post-operatively. Among iatrogenic ureteral injuries in gynaecology, most occur in hysterectomy (54\%), pelvic operations such as ovarian tumour removal (8\%), and transabdominal urethropexy (8\%) [1-3, 8-10]. The leading cause of ureteral injury in laparoscopic surgery is vaginal hysterectomy (20\%), resection of endometriosis (12.8\%), oophorectomy (11.4\%), pelvic lymphadenectomy $(10 \%)$, sterilization (7.1\%), and adhesiolysis and drainage of lymphocele (4.3\%) [5]. The experience of the surgeon plays a crucial role in the incidence of iatrogenic ureteral injury. Rates of ureteral trauma vary form $0.3 \%$ for experienced gynaecologists to $14 \%$ for those with little experience $[3,6]$.

\section{Case report}

A 65-year-old woman with multiple uterine myomas and right ovarian cystic lesion underwent a laparoscopic subtotal hysterectomy with bilateral salpingoophorectomy at a district gynaecology department. Two weeks later she noted a urine leakage from the vagina, which is a symptom requiring careful evaluation in the post-operative period [11]. On admission, pelvic examination revealed a urinary fistula in the vagina (Fig. 1) with continuous urine leakage. A computed tomography (CT) scan revealed mild dilation of the left renal pelvis and the upper two thirds of the ureter due to an inflammatory fibrous mass with air bubbles (Fig. 2) involving its lower part. Contrast enhancement identified the site of urine leakage (Figs. 3-5). Urological examination with cystoscopy and ureteroscopy (URS) revealed discontinuity in the lower third of the left ureter with a fibrous mass in the lumen (Fig. 6). An attempt to place a double-J stent in the left ureter failed. Considering the results of com- 


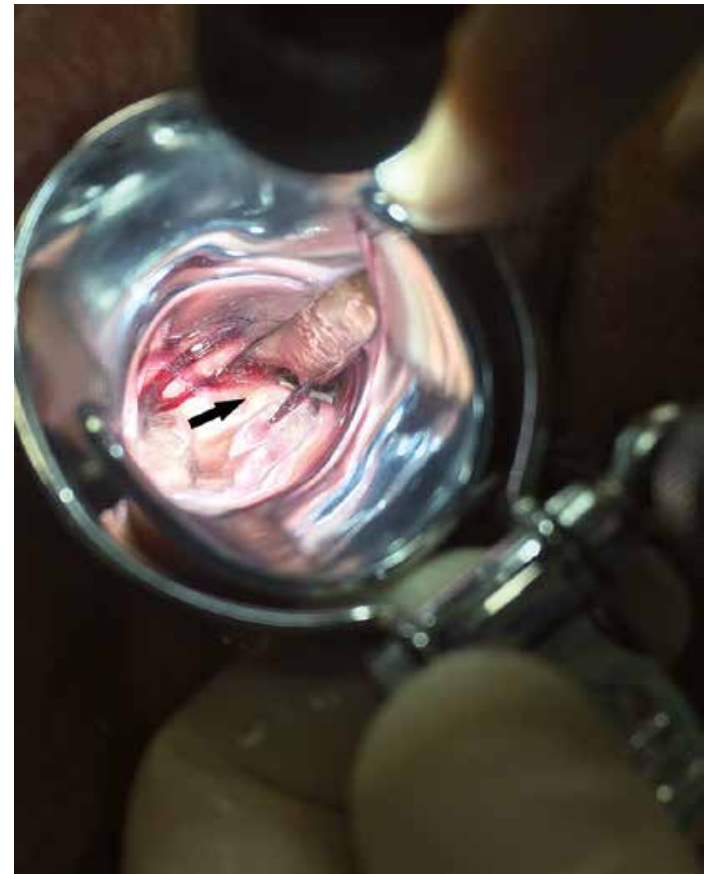

Fig. 1. Left ureterovaginal fistula in vaginal examination

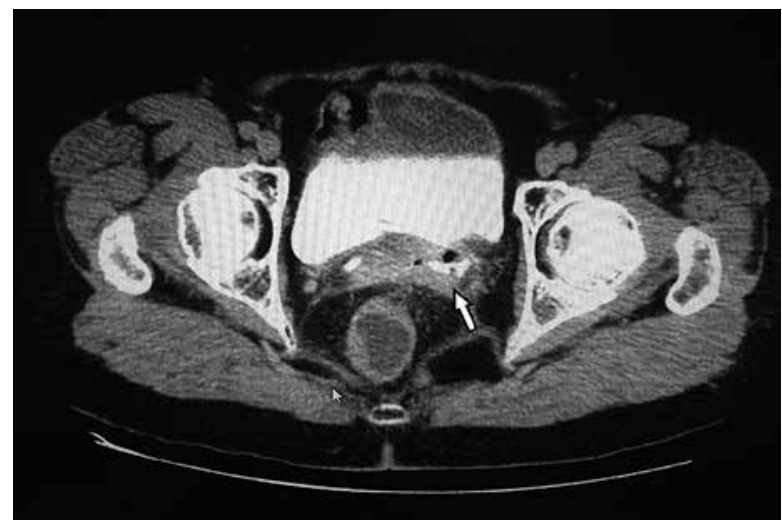

Fig. 4. Contrast-medium leakage from left ureter in the site of inflammatory fibrous mass

puted tomography and ureteroscopy, classic laparotomy with ureteral repair or ureteroneocystostomy was chosen as a method of treatment. After a month the patient was admitted to the urology department and underwent a laparotomy with left ureteroneocystostomy (Figs. 7-9). During the operation the left ureter was mobilized from surrounding tissues along the entire length up to the crossing with the left upper bladder artery. Intraoperatively, involvement of the ureter in a fibrous mass extending into the perivesicular space was revealed. It was not possible to mobilize the ureter from the stiff mass. The ureter was cut at the most distal point to the fibrous tissue. The distal ureteral cuff was sewn and ligated with Vicryl 2/0 and left in the mass. A double-J stent 4.7Fr was inserted into the left kidney pelvis via the dilated proximal ureteral cuff. The left

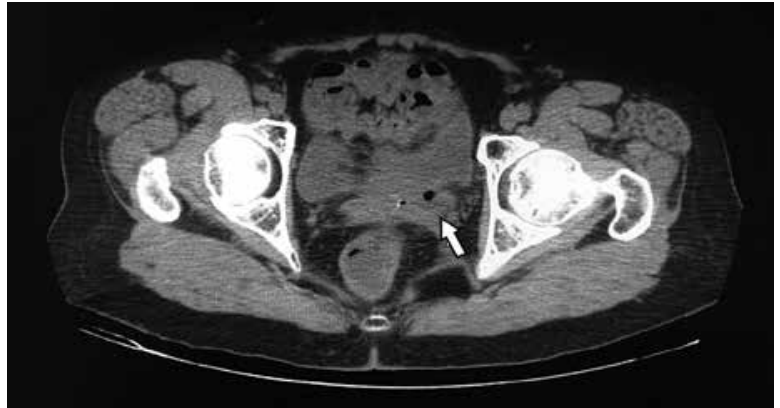

Fig. 2. Postoperative inflammatory fibrous mass with air bubbles in computed tomography of the pelvis

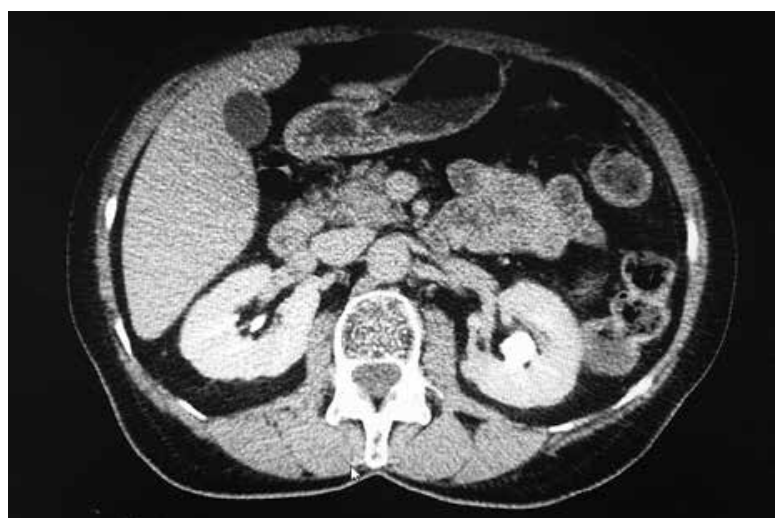

Fig. 3. Left ureterohydronephrosis in computed tomographyurography

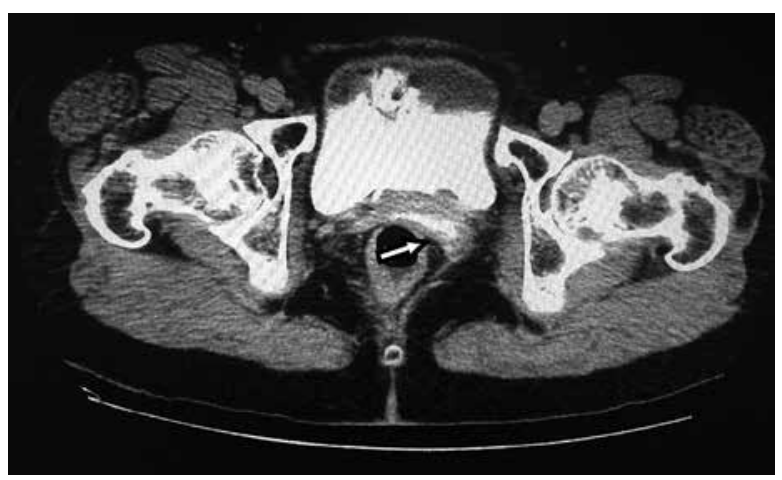

Fig. 5. Contrast-medium leakage from left ureter to vagina

upper bladder artery was ligated, then the ureter was reimplanted in the left lateral bladder wall. Ureteroneocystostomy was made using the Lich-Gregoir technique over a double-J stent. The bladder wall was closed in 2 layers, and finally a transurethral catheter was inserted. The post-operative period was uncomplicated. After 14 days, the transurethral catheter was removed and the patient passed urine without leakage. The double-J stent was removed after 4 weeks. The follow-up after 3 months revealed no signs of left hydronephrosis on ultrasonography check. During vaginal examination there was no vaginal urine leakage, wound margins were covered with epithelium, and the fistula bed was filled with granular tissue. 


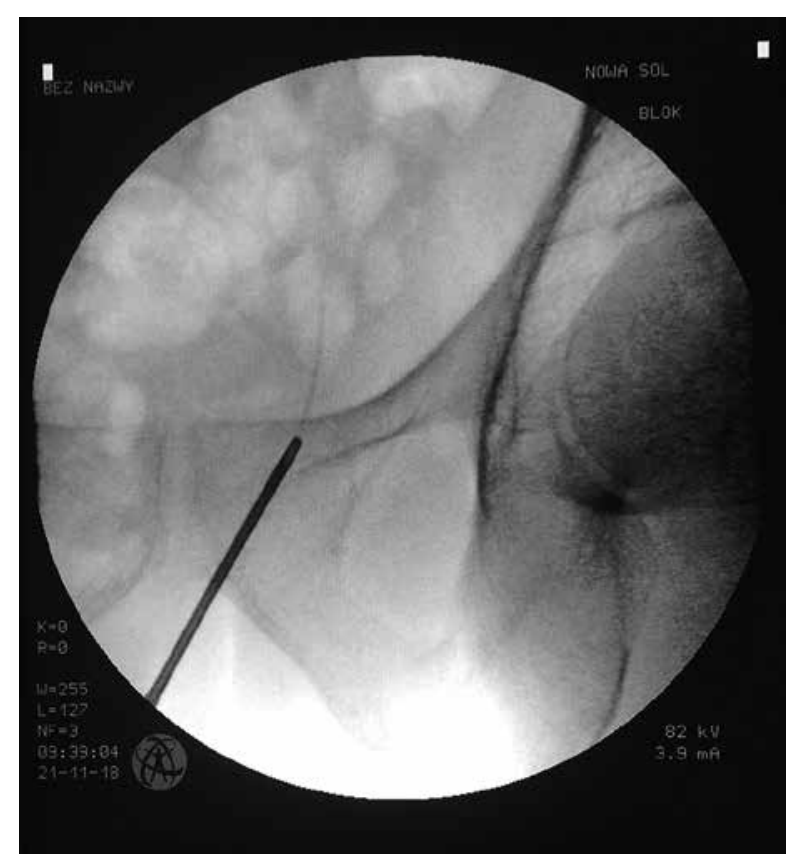

Fig. 6. X-ray during URS showing the site of ureteral injury

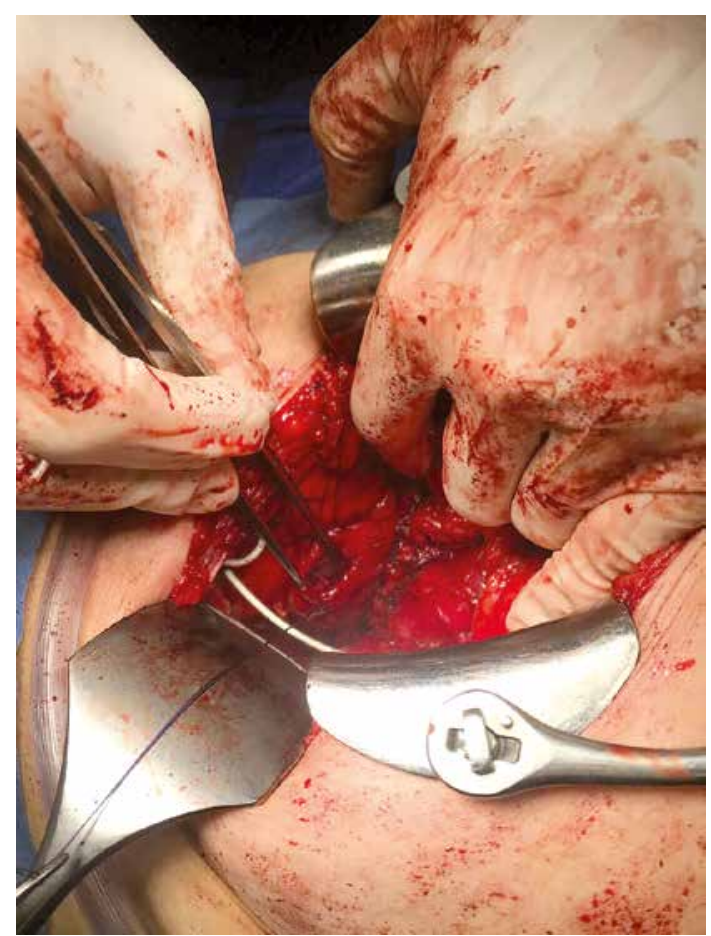

Fig. 8. Cystotomy in the site of lateral bladder wall and distal end of Double-J stent placement

\section{Discussion}

The mechanisms of ureteric injuries during operative procedures include crushing with a clamp, suture ligation, partial or complete primary transection, angulation, avulsion, or ischaemic necrosis following electrocoagulation. Significant complications after ureteral injury include renal insufficiency, ureteral strictures,

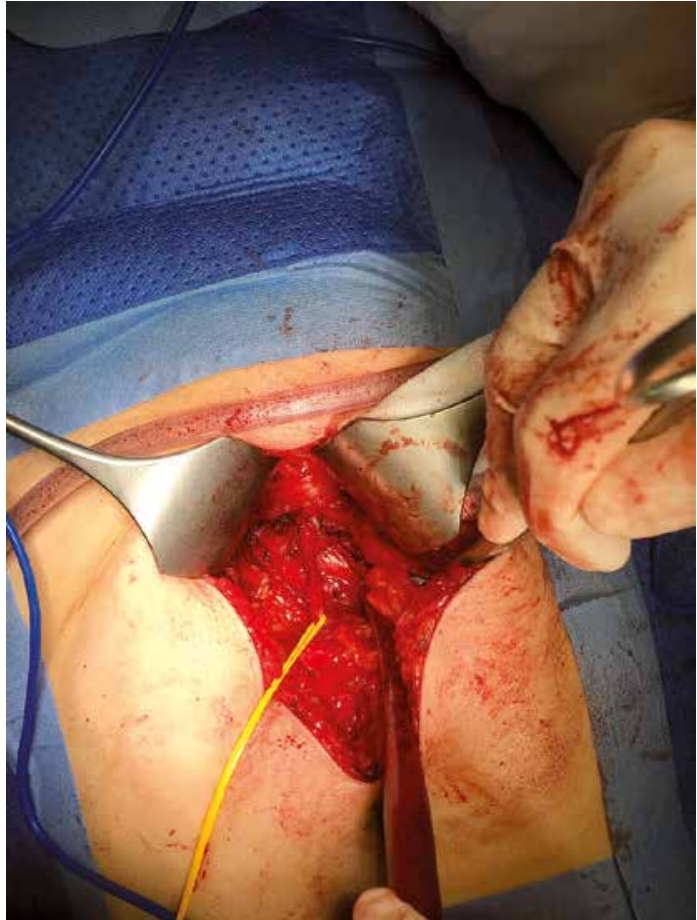

Fig. 7. Mobilized left ureter up to the site of involvement in the fibrous mass

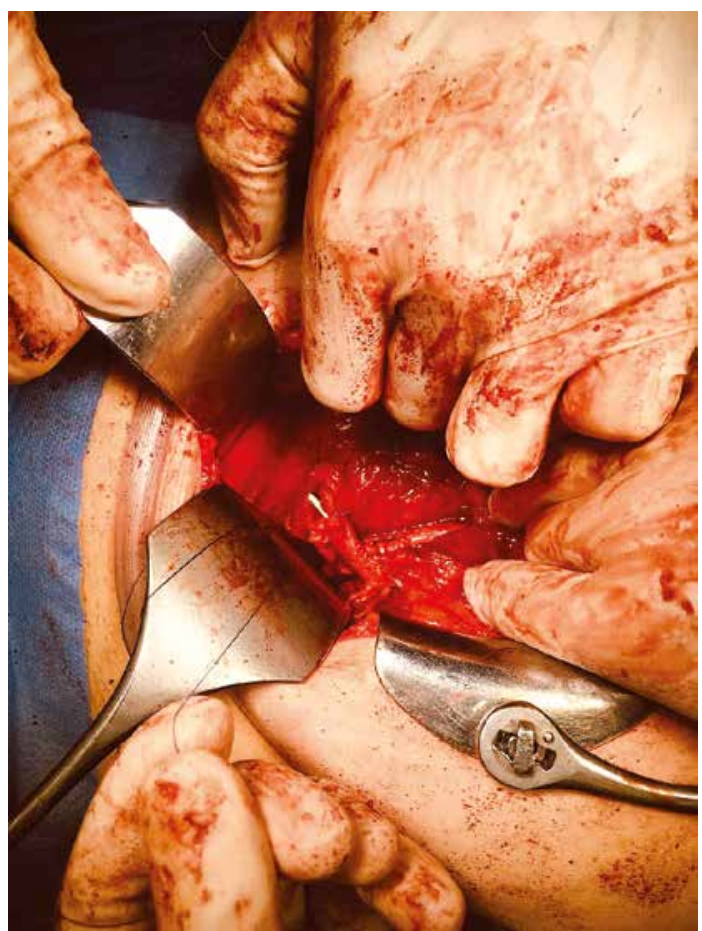

Fig. 9. Left ureteroneocystostomy using Lich-Gregoir technique over double-J stent

vesicovaginal, and ureterovaginal fistulae. Ureteral fistulas to the genital tract in females may connect with the vagina or much less commonly with the fallopian tube or the uterus [7]. Risk factors for the development 
of ureterovaginal fistula include endometriosis, obesity, pelvic inflammatory disease, as well as radiation therapy and pelvic malignancy. The main presentation of the ureterovaginal fistula is urinary incontinence despite the normal act of micturition [8]. Incontinence usually begins between 1-4 weeks after surgery. Initially, the patient may experience flank pain, fever, and nausea due to the urinoma or obstructed kidney, followed by incontinence. Reviewed studies [9] considered ultrasonography, intravenous urography, retrograde pyelography, cystography, CT-urography, MRI, 3-swab test, and vaginal examination useful for the diagnosis of ureteral obstruction and ureterovaginal fistula. Cystogram may be useful to exclude a coexistent vesicovaginal fistula. The incidence of left-side ureteral fistula is $88.2 \%$ as compared to $11.7 \%$ on the right side $[4,8]$. The probable reason is the fact that the operating gynaecologist usually stands on the right side of the patient; hence,the left-sided bleeding is controlled under obscured vision. The goal of the treatment of ureterovaginal fistula is the resolution of urinary leakage, prevention of urosepsis, and preservation of renal function. Early drainage of the affected upper urinary tract is essential [5]. Immediate open surgical repair may be difficult; therefore, ureteral stenting or percutaneous nephrostomy are feasible options. Endoscopic management with ureteral stenting can promote closure of the fistula if initiated early. Persistent urinary leakage can be treated with percutaneous nephrostomy drainage, ureteral stent(s), and/ or Foley catheter drainage. In more complex injuries treatment includes open surgeries and minimally invasive laparoscopic or robotic reconstructive surgeries, which involve ureteroureterostomy, ureteroneocystostomy, and ureteral replacement using ileal interposition or autografts. The majority of ureterovaginal fistulas develop due to injuries in the distal third of the ureter, below the pelvic brim, and can be repaired with ureteroneocystostomy. The Lich-Gregoir ureteroneocystostomy technique is most commonly used. There was no difference in outcome between refluxing and non-refluxing implantation in adults. In the majority of cases ureteroneocystostomy is combined with a psoas hitch manoeuvre or a Boari flap in order to cover a greater distance and facilitate tension-free anastomosis [5]. In cases of bilateral or combined fistulas of ureterovaginal and vesicovaginal fistula, management may require bladder reconstruction or urinary diversion.

Significant preventive measures for surgeons are as follows: 1) proper evaluation of surgical indications for hysterectomy and consideration of other treatment options; 2) experience using laparoscopic and laparotomy techniques, knowledge of all risks and benefits of each method; 3) knowledge of anatomical landmarks: location of blood vessels, nerves, ligaments, careful identification of the ureter; 4) knowledge of electrosurgery principles: depth, penetration, spread; 5) adequate exposure during operation: pneumoperitoneum, Trendelenburg position, bowel preparation, immediate control of bleeding, cautious tissue dissecting, intrauterine manipulator if needed; 6) preoperative recognition: cystoscopy with intravenous injection of indigo carmine or placement of ureteral stents; 7) strict monitoring in every case: temperature, passage of gas and stool, loin pain, vaginal leakage, serum C-reactive protein, and creatinine levels.

\section{Conclusions}

The morbidity associated with ureteral injury may be serious, resulting in prolonged hospital stay, suboptimal surgical outcome, secondary invasive interventions, decreased renal function and reduction of the patient's quality of life. The risk of ureteral complications after laparoscopic hysterectomy is comparable to open laparotomy. Surgical expertise, knowledge of pelvic wall anatomy, and careful identification and mobilisation of the ureter are the key measures to prevent injury. Early diagnosis is crucial to prevent long-term treatment. Endoscopic techniques are sufficient in the majority of early diagnosed cases. Nevertheless, more extensive reconstructive surgery is needed in ureteral injuries developing into renal insufficiency, urogenital fistulas, and severe ureteral strictures.

\section{Disclosure}

The authors report no conflict of interest.

\section{References}

1. Patil SB, Guru N, Kundargi VS, et al. Posthysterectomy ureteric injuries: presentation and outcome of management, Urol Ann 2017; 9: 4-8.

2. Wijaya T, Lo TS, Bin Jaili S, et al. The diagnosis and management of ureteric injury after laparoscopy. Gynecol Minimall Invasive Ther 4, 2015: 29-32.

3. Vasavada SP, Fields Schwartz B. Ureteral injury during gynecologic surgery. https://emedicine.medscape.com/article/454617-overview.

4. Hanif MS, Saeed K, Sheikh MA. Surgical management of genitourinary fistula. J Pak Med Assoc 2005; 55: 280-284.

5. Gild P, Kluth LA, Vetterlein MV, et al. Adult iatrogenic ureteral injury and stricture incidence and treatment strategies. Asian J Urol 2018; 5: 101-106.

6. Leonard F, Fotso A, Borghese B, et al. Ureteral complications from laparoscopic hysterectomy indicated for benign uterine pathologies: a 13-year experience in a continuous series of 1300 patients. Hum Reprod 2007; 22: 2006-2011

7. El-Agwany AS. A case study regarding iatrogenic ureterovaginal fistula following hysterectomy: radiologic findings. Arch Perin Med 2014; 20 : 176-178.

8. Murtaza B, Mahmood A, Azim Niaz W, et al. Ureterovaginal fistula etiological factors and outcome. J Pak Med Assoc 2012; 62: 999-1003.

9. Mandal AK, Sharma SK, Vaidyanathan S, et al. Ureterovaginal fistula: summary of 18 years experience. Br J Urol 1990; 65: 453-456.

10. Randawa A, Khalid L, Abbas A. Diagnosis and management of ureterovaginal fistula in a resource-constrained setting: experience at a district hospital in northern Nigeria. Libyan J Med 2009; 4: 41-43.

11. La Rosa VL, Platania A, Ciebiera M, et al. A comparison of sacral neuromodulation vs. transvaginal electrical stimulation for the treatment of refractory overactive bladder: the impact on quality of life, body image, sexual function, and emotional well-being. Prz Menopauzalny 2019; 18 : 89-93. 